\title{
A Microwave Band Pass Filter with Suspended Diamond Slot Structure
}

\author{
Tiandi Xie, Mingzhe Hu* \\ Department of Physics and Electronics, Liupanshui Normal University, Liupanshui 553004, Guizhou, \\ China \\ *Corresponding author: mingzhe-hu@163.com
}

Keywords: Spoof SPPs, microwave filter, diamond slot, computer simulation, band pass filter

\begin{abstract}
In the present paper a band pass microwave filter based on spoof surface plasmon polaritions (spoof SPPs) mechanism and with suspended diamond slot structure is designed and simulated. The filter is composed of three sections, where in the third section, a corrugated metallic strip with parallel and periodically arranged diamond slots is the spoof SPPs transmission line. In the second section, the transition section, the gradient diamond slots are designed and distributed with the same periodic as the spoof SPPs section to realize the minimum transmission impedance. The transmission and reflection properties of the spoof SPPs filter in microwave frequency region are elaborately investigated. Through adjusting the geometrical dimensions of the suspended diamond-shaped slot structure, the bandwidth and suppression characteristics of the filter can be flexibly controlled and the filter has great resistant ability to space electromagnetic interference.
\end{abstract}

\section{Introduction}

The surface plasmon polaritons (SPPs) is a kind of surface electromagnetic wave. It propagates along the interface between the metal and the dielectric interface with an amplitude decaying exponentially in the direction vertical to the interface, since the metal has the similar property to plasma with a negative permittivity [1]. SPPs have been proposed to transform the traditional Sommerfeld or Zenneck surface waves into the highly confined electromagnetic waves, which provide favorable conditions to overcome the diffraction limit [2]. Therefore, SPPs have been researched in areas of super-resolution imaging [3], electromagnetically induced transparency (EIT) [4], energy harvesting [5] and SPP circuits [6]. However, natural SPPs effect can only work at optical frequency since the intrinsic electron oscillation in a metal is usually located beyond the infrared band. While at much lower frequency band, which is actually the microwave and terahertz communication frequency bands, deep sub-wavelength effect of SPPs cannot be realized because the metal behave assemble as perfectly electrical conductor (PECs), with large imaginary and negative real part of the permittivity, disabling the internal plasmonic oscillations in metal.

With the development of SPP devices, in 2004, professor Pendry et al. put forward a device, which is the so-called spoof SPPs concept. The spoof SPPs device can produce deep sub-wavelength effect at microwave and terahertz frequencies, which can be employed to fabricate novel microwave compact devices [7]. The spoof SPPs microwave device, compared with traditional microwave devices, possesses special properties [8-11]. For example, it can confine the microwave field into sub-wavelength size, it possesses better resistibility to electromagnetic interference as well as possesses higher sensitivity and larger bandwidth. Therefore, the filters based on the spoof SPPs can be very suitable to meet the requirements of the next generation microwave communication [12-13].

Based on the above technology background, in the present paper, a novel kind of spoof SPPs band pass microwave filter is designed and investigated. The spoof SPPs are generated by artificial suspended diamond-shaped slots with periodic arrangement, which can improve the sub-wavelength confinement effect in microwave band, so that the spoof SPPs filter has better suppression characteristics. 


\section{Filter Design Theory}

A novel kind of spoof SPPs filter working in microwave frequency range is designed, which consists of three parts. The first part is the microstrip waveguide with the length of 11 , realizing the input or output of the microwave signal, the second one is the microwave mode conversion section with the length being 12, the microstrip waveguide in the first part working in quasi TEM mode will be smoothly converted into the SSPPs mode in the second part, where, a novel periodically arranged suspended diamond-shaped slot structure is designed. These suspended diamond-shaped slots can enhance the confinement effect of microwave band sub-wavelength so as to improving the stop-band characteristics. Additionally, it can improve the ability of resisting electromagnetic interference of the spoof SPPs filter. By adjusting the geometrical dimensions of the suspended diamond structure in section 3, we can precisely control the bandwidth and suppression characteristics of the filter. Especially, the suspended diamond slot structure does not increase the overall geometric size of the spoof SPPs transmission lines whilst optimizes the filtering characteristics of the bandpass filter.

Finally, in the back side of the filter, a metal ground is designed which possesses the curve of elliptic equation. The elliptic equation can be described as:

$$
\frac{\left(x-l_{1}-l_{2}-l_{3} / 2\right)^{2}}{\left(l_{1}+l_{2}+l_{3} / 2\right)^{2}}+\frac{(y+h+w)^{2}}{a^{2}}=1
$$

where a is the radius of short axis of elliptic curve, $h$ is the width of the metal microstrip, $w$ is the position coefficient of elliptic curve. The overall geometric size of the filter, including the front side and back side, is illustrated in Fig. 1 and the physical dimension of each part of the filter is listed in Table 1.

TABLE 1 The physical dimensions of each part of the microwave filter

\begin{tabular}{ccc}
\hline Structure & Symbol & Size \\
\hline Microstrip waveguide length & $\mathrm{l}_{1}$ & 10 \\
Transition length & $\mathrm{l}_{2}$ & 60 \\
Spoof SPPs segment length & $\mathrm{l}_{3}$ & 103 \\
Radius of short axis of elliptic curve & $\mathrm{a}$ & 5 \\
Width of suspended diamond slot I & $\mathrm{w}_{1}$ & 1.0 \\
Width of suspended diamond slot II & $\mathrm{w}_{2}$ & 3.0 \\
Length of suspended diamond slot & $\mathrm{l}_{4}$ & 16.0 \\
side gap of diamond slot with the & $\mathrm{d}$ & 0.8 \\
microstrip & & \\
The periodic of the diamond slots & $\mathrm{p}$ & 5.0 \\
Elliptic curve position coefficient & $\mathrm{w}$ & 25 \\
Microstirp width & $\mathrm{h}$ & 20 \\
Dielectric substrate width & $\mathrm{w}_{\text {sub }}$ & 30.3 \\
\hline
\end{tabular}

\section{Numerical Results and Discussion}

A substrate material with $0.5 \mathrm{~mm}$ thickness and permittivity of 2.65 and loss tangent of 0.003 when working at microwave frequency is employed for the spoof SPPs filter. The filtering characteristic curve is calculated and the numerical results are demonstrated in Fig. 2. The filter is a band pass filter with its center frequency of $4.997 \mathrm{GHz}$. The insertion loss at the center frequency is $-1.7 \mathrm{~dB}$. The band pass width of the $-3 \mathrm{~dB}$ insertion loss is in the range of $0.661 \mathrm{GHz}$ to $8.333 \mathrm{GHz}$ with the relative bandwidth being 
larger than $150 \%$. The reflection coefficient $\mathrm{S}_{11}$ in the whole pass band is less than $-7.8 \mathrm{~dB}$ and the ripple in the whole pass band is less than $1.3 \mathrm{~dB}$, as illustrated in Fig. 2.

To get a direct physical insight into the electromagnetic wave mode matching effect, as well as the properties of field propagation and the confinement on the spoof SPPs waveguide with the suspended diamond slot structure, the energy flows (on a dB scale) at $6.0 \mathrm{GHz}$ toward $\mathrm{x}$ direction on the xoy plane that is $0.5 \mathrm{~mm}$ above the plasmonic surface of the waveguide is exhibited in Fig. 3. It is obviously observed that the electromagnetic wave transmits through the filter with low reflection. The EM energy is tightly confined in deep sub-wavelength scale around the plasmonic waveguide and it propagates with low absorption in the whole pass band.

Moreover, the stop band can be precisely controlled by the suspended diamond slot parameter in the third section. Additionally, the transition section plays an important role in the impedance matching between the first semi TEM section and the third spoof SPPs section, as illustrated in Fig. 2(b). Without the transition section, the reflection of the filter increases much larger and the transmission coefficient decreases obviously, which indicates that the transition section transfer the electromagnetic mode and impedance smoothly.

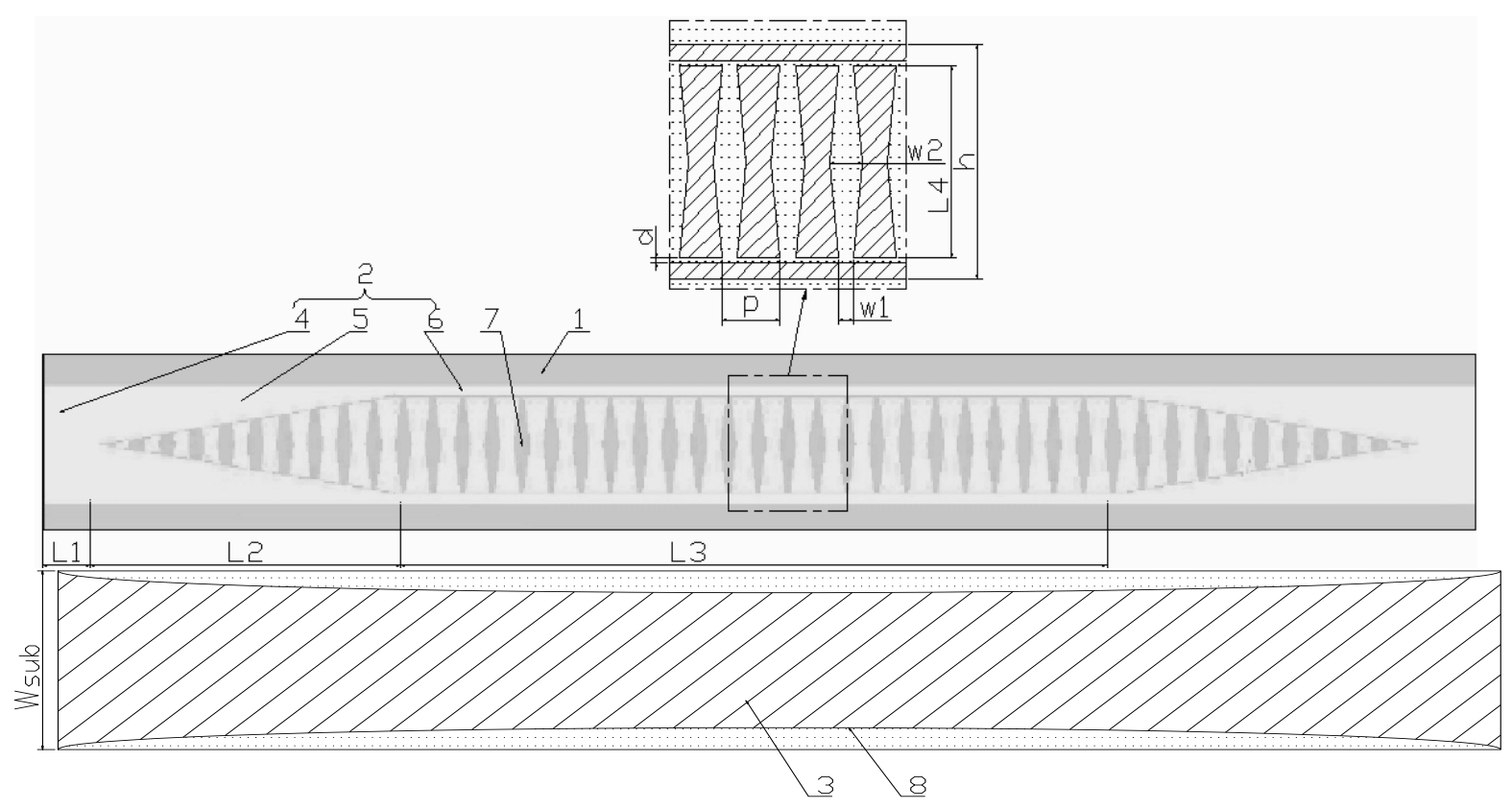

Fig. 1 The geometric structure of the proposed spoof SPPs filter. The above figure is the front side of the filter, where 1 is the woven-glass substrate, 2 is the microstrip metal, 4 is the microstrip waveguide, 5 is the transition section, 6 represents the SSPPs waveguide section, 7 shows the suspended diamond slots in the SSPPs section, 3 is the ground metal with elliptic curve 8 in the microwave filter. The below figure is the back side of the filter, where, the oblique line is the the ground in the elliptic curve shape. 

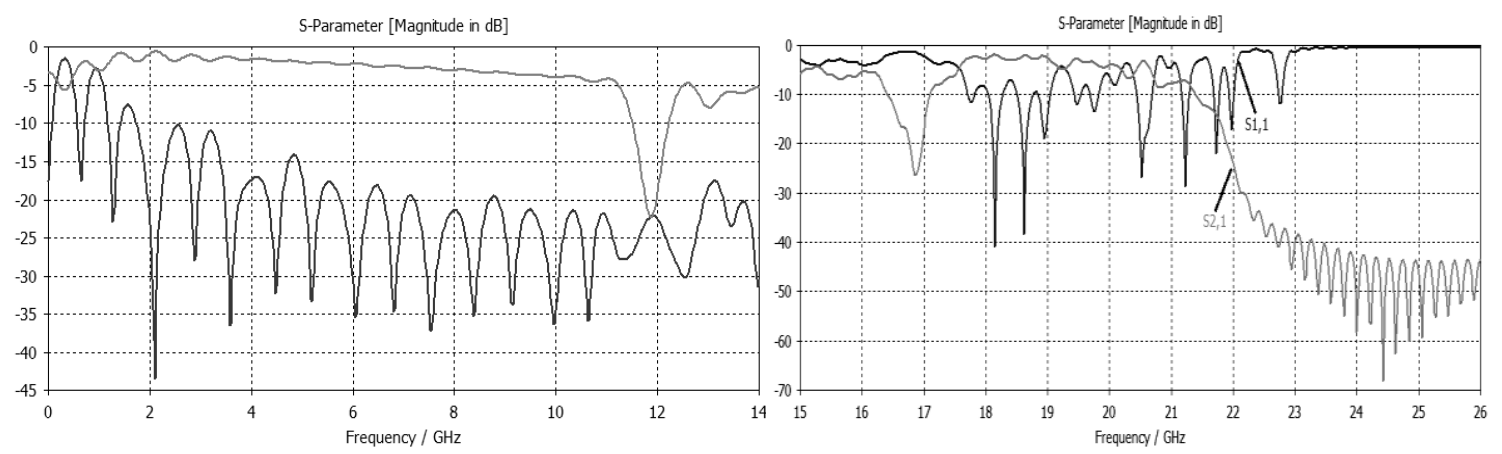

Fig. 2 The simulated S-parameters of the presented SPPs filter (with transition section (a), without transition section (b)) with the geometric structure being listed in Table 1 and figure 1.

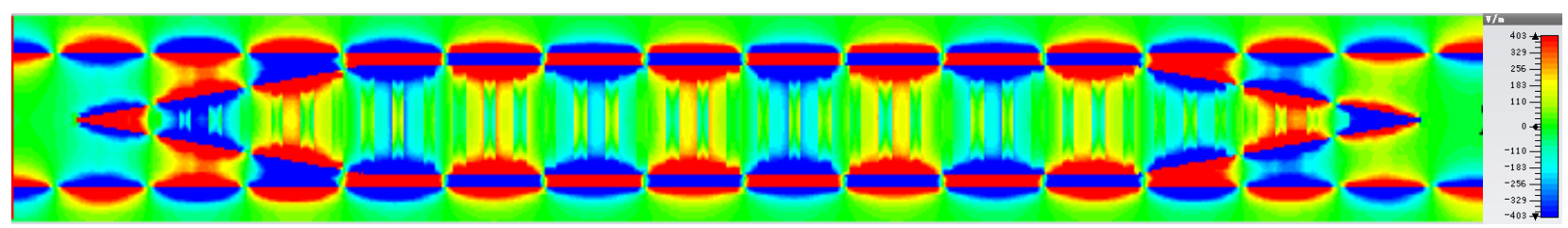

Fig. 3 The simulated E field of the presented filter at $6.0 \mathrm{GHz}$ working frequency.

\section{Summary}

A novel microwave band pass filter with spoof SPPs structure is designed and investigated in the present paper. The microwave filter consists of three sections, where in the second conversion section, the TEM mode is smoothly converted into the SSPPs mode. The SSPPs mode is generated by a novel periodically arranged suspended diamond slot structure, which can enhance the confinement effect of electromagnetic wave into sub-wavelength as well as improve the stop band characteristics of the microwave filter. Also, the suspended diamond slot structure can improve the ability of resisting electromagnetic interference of the filter. With the optimal parameters presented, the filter can work at the center frequency of $4.997 \mathrm{GHz}$ with the insertion loss being $-1.7 \mathrm{~dB}$ at this point. The $-3 \mathrm{~dB}$ bandwidth of the filter is in the range of $0.661 \mathrm{GHz}$ to $8.333 \mathrm{GHz}$ with the relative bandwidth of being larger than $150 \%$. The reflection in the whole pass band is less than $-7.8 \mathrm{~dB}$ and the ripple in the whole pass band is less than $1.3 \mathrm{~dB}$. The bandwidth of the present filter can be precisely controlled by the geometre of the suspended diamond slot structure in the third section. These properties manifest that the filter possesses great penitential applications in the modern microwave communication systems.

\section{Acknowledgement}

The present work is partially supported by the National Science Foundation of China (Nos.51567017), partially supported by Physical Electronic Key Discipline of Guizhou Province (No. ZDXK201535), partially supported by the Key Project of the Education Department of Guizhou Province(No.2013174), partially supported by the Outstanding Young Scientist Cultivation Program of Guizhou Province (No.201522), partially supported by the Joint Science Foundation of Liupanshui City and Guizhou Province (Nos. LH20147449), partially supported by the Key Laboratory of Opt-electrical Information Technology of Liupanshui City (No.52020201420205), partially supported by the MIT Creative Team of Liupanshui Normal University (No.LPSSYKJTD201402), partially supported by the Recruitment Program of Liupanshui Normal University (No. LPSSYKYJJ201403) and partially supported by the 
Key Laboratory of the Reliability of Power Components and Devices of Guizhou Province (Grant No. KFJJ201505).

\section{References}

[1] Barnes, W. L., Dereux, A. \& Ebbesen, T. W. Surface plasmon subwavelength optics. Nature 424, 824-830, 2003.

[2] Gramotnev, D. K. and S. I. Bozhevolnyi, "Plasmonics beyond the diffraction limit," Nat. Photonics, Vol. 4, 83-91, 2010.

[3] Lu, D. and Z. Liu, "Hyperlenses and metalenses for far-field super-resolution imaging," Nat. Commun., Vol. 3, 1205, 2012.

[4] Han, Z. and S. I. Bozhevolnyi, "Plasmon-induced transparency with detuned ultracompact FabryPerot resonators in integrated plasmonic devices," Opt. Express, Vol. 19, 3251- 3257, 2011.

[5] Linic, S., P. Christopher, and D. B. Ingram, "Plasmonic-metal nanostructures for efficient conversion of solar to chemical energy," Nat. Mater., Vol. 10, 911-921, 2011

[6] Zhao, C. and J. Zhang, "Plasmonic demultiplexer and guiding," Acs Nano, Vol. 4, 6433-6438, 2010.

[7] Pendry J., Martin M., Garcia-Vidal F. L., Mimicking surface plasmons with structured surfaces. Science 305, 847-848, 2004.

[8] Anker J. N., Hall W. P., Dres O. L., Shan N. C., Zhao J., \& Duyne R. P. V., Biosensing with plasmonic nanosensors. Nature Mater. 7, 442-453, 2008.

[9] Jones A. C., Olmon R. L., Skrabalak S. E., Wiley B. J., Xia Y. N., \& Raschke M. B. Mid-IR plasmonics: near-field imaging of coherent plasmon modes of silver nanowires. Nano Lett. 9, 2553 2558, 2009.

[10] Fang N., Lee H., Sun C., \& Zhang X., Sub-diffraction-limited optical imaging with a silver superlens. Science 308, 534-537, 2005.

[11] Pendry J., Playing tricks with light. Science 285, 1687-1688, 1999.

[12] Yin J. Y., Zhang H. C., Fan Y. F., \& Cui T. J., Direct radiations of surface plasmon polariton waves by gradient groove depth and flaring metal structure. IEEE Antennas Wireless Propag. Lett. $15,865-868,2015$

[13] Pan B. C., Liao Z., Zhao J., \& Cui T. J., Controlling rejections of spoof surface plasmon polaritons using metamaterial particles. Opt. Exp. 22, 13940-13950, 2014. 